\title{
HIGH LUMINOSITY OPERATION OF THE FERMILAB ACCELERATOR COMPLEX*
}

\author{
S. Mishra ${ }^{\#}$, Fermilab, Batavia, IL 60510, USA
}

\begin{abstract}
Run-II at Fermilab is progressing steadily. In the Run-II scheme, 36 antiproton bunches collide with 36 proton bunches at the CDF and D0 interaction regions in the Tevatron at $980 \mathrm{GeV}$ per beam. The current status and performance of the Fermilab Accelerator complex is reviewed. The plan for Run-II, accelerator upgrades and integration of the Recycler in the accelerator chain will be presented.
\end{abstract}

\section{INTRODUCTION}

The Fermilab is the highest energy colliding beam accelerator in the world. It will remain so till the turn-on of the LHC. The accelerator complex is running with the new Main Injector and upgraded proton source that consists of the Linac and Booster, an upgraded Antiproton source, and the Tevatron. The commissioning of the Recycler continues. The collider physics program is making steady progress with two upgraded detectors and increasing luminosity. This paper will give a brief introduction to the accelerator complex and, present the performance, issues and upgrade plans for the accelerators. We will also present the collider performance and exciting physics results from the two experiments.

\section{ACCELERATOR COMPLEX}

Figure 1 shows the schematic view of the Fermilab accelerator complex with the newly commissioned Main Injector and Recycler. The proton production cycle starts at the Crockoft-Walton. The Crockoft-Walton accelerates $\mathrm{H}^{-}$ions to $750 \mathrm{KeV}$ and injects them into the Linac. The linac accelerates the $\mathrm{H}^{-}$ions to $400 \mathrm{MeV}$. The $\mathrm{H}^{-}$ions are stripped and injected into the Booster. Protons are accelerated in the Booster to $8.9 \mathrm{GeV} / \mathrm{c}$ in $33 \mathrm{msec}$ and transferred to Main Injector using an $8.9 \mathrm{GeV} / \mathrm{c}$ transfer line built using permanent magnets. In the Main Injector the protons are accelerated from $8.9 \mathrm{GeV} / \mathrm{c}$ to $120 \mathrm{GeV} / \mathrm{c}$ in $1.5 \mathrm{sec}$ and sent to the pbar production target. For Tevatron operation the Main Injector accelerates 5-7 bunches of protons to $150 \mathrm{GeV} / \mathrm{c}$, coalesces them into one bunch, and transfers the bunch to the Tevatron.

At present, Main Injector accelerates about $5 \times 10^{12}$ protons to $120 \mathrm{GeV} / \mathrm{c}$ every $2.4 \mathrm{sec}$ and extracts it to a nickel production target. A lithium lens operating at 760 Tesla/meter downstream of the antiproton production target focuses $85 \times 10^{6}$ antiprotons at $8.9 \mathrm{GeV} / \mathrm{c}$. These antiprotons are collected, stored and stochastically cooled in the Debuncher and Accumulator (using the stacking lattice). When stack reaches somewhere between 100 $150 \mathrm{e} 10$ anti-protons and the Tevatron is ready for the next store, the $8.9 \mathrm{GeV} / \mathrm{c}$ antiprotons are extracted from the
Accumulator (using the shot lattice), and injected in to the Main Injector. The Main Injector accelerates the antiprotons to $150 \mathrm{GeV}$ using the $53 \mathrm{MHz}$ rf system, coalesces them, and transfers them to the Tevatron.

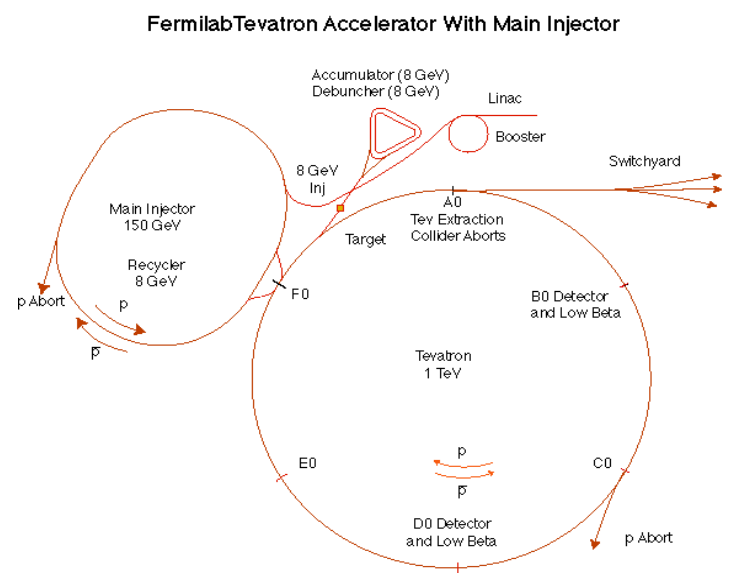

Figure 1. Schematic layout of the Fermilab Accelerator complex.

The Tevatron accelerates both protons and antiprotons from $150 \mathrm{GeV}$ to $980 \mathrm{GeV}$ on helical orbits and collides them at the B0 and D0 interaction regions.

Major changes to accelerator between Run-I and Run-II are: a) Rapid cycling Main Injector replaced Main Ring, b) Antiproton source and Tevatron were upgraded to handle higher intensity, c) the number of proton and pbar bunches have been increased from 6 to 36, with a crossing time of $396 \mathrm{nsec}$, d) the Tevatron collision energy has increased from $1.8 \mathrm{TeV}$ to $1.96 \mathrm{TeV}$, and e) The Recycler a new permanent magnet $8.9 \mathrm{GeV} / \mathrm{c}$ storage ring for antiproton accumulation was added and is now being commissioned.

\section{PROTON SOURCE}

The Proton Source is made up of the Crockoft-Walton, Linac, and Booster. The performance of these accelerators is excellent and the Booster is accelerating higher intensity proton beams to $8.9 \mathrm{GeV} / \mathrm{c}$ for Run-II and the MiniBooNE experiment [1]. Figure 2 show the intensity and beam loss plot for the $33 \mathrm{msec}$ Booster acceleration cycle. There is a loss of beam in first few msec at the injection energy and early in the acceleration cycle. This loss is attributed to space charge effects, momentum spread and lattice issues. There is a small loss of beam at transition energy as shown by an increase in the loss monitor readings (red curve in Figure 2).

Linac and Booster are routinely delivering 250k pulses in 24 hours with an average repetition rate of $3 \mathrm{~Hz}$ and 
peak rate of $5 \mathrm{~Hz}$. The total intensity delivered is being limited by losses, which lead to activation in the Booster tunnel. Booster delivers 4-5e16 protons per hour to users.

Physics issues that are being addressed includes a) the space charge effect at injection, b) emittance preservation through the ramp, and c) better understanding of the injection lattice.

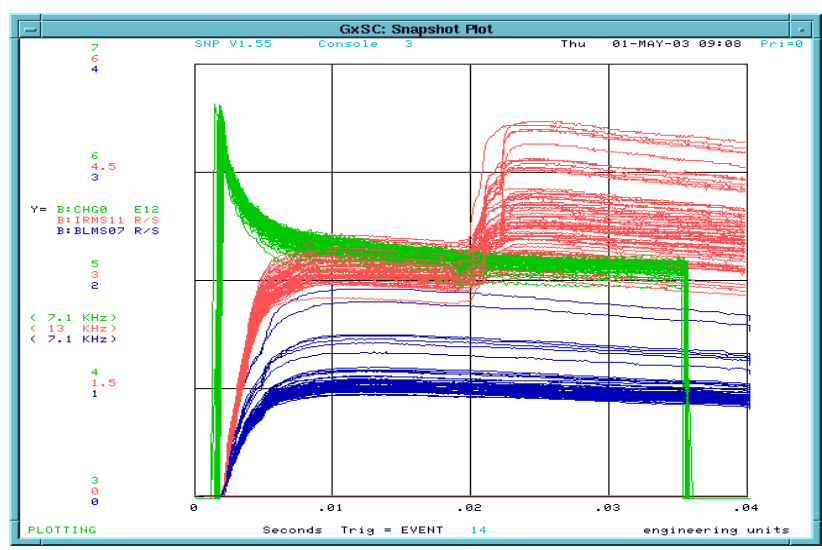

Figure 2. Beam intensity and losses through the Booster acceleration cycle. Green line is proton intensity, blue and red are loss monitor readings.

The optics of the Booster is significantly perturbed by the edge focusing of the injection and extraction orbit bumps [2]. There is a sizable dispersion error at injection in the Booster. It has been calculated and measured that the injection "Or-Bump" and the "dog-leg" around the extraction septum is contributing to this error.

Increasing the proton intensity and reducing the loss of beam in the Booster tunnel is the main emphasis of the Proton Source Department. Several projects including a) Longitudinal Dampers, b) transition jump system, c) ramped corrector to control the orbit and improve aperture, d) ramp monitor program, and e) larger aperture RF cavities.

\section{MAIN INJECTOR}

The Main Injector was commissioned in 1999 and has been supporting the Fermilab physics program since that time. Main Injector has achieved most of its design goals and the main emphasis continues to be on improving the quality of beam [3]. Recent development includes a) lattice matching between injection and extraction lines to Main Injector to preserve emittances, b) beam loading compensation for proton coalescing, c) multi-batch beam loading compensation for antiproton coalescing, d) R\&D on a longitudinal damper to reduce the longitudinal emittance growth in the Main Injector, e) development of transverse dampers for injection and high intensity operation, f) development of a $2.5 \mathrm{MHz}$ acceleration scheme for Recycler antiproton acceleration, g) an upgraded BPM system and h) development of slip stacking to increase the proton flux on the antiproton production target. Main Injector runs more than 95\% efficient for pbar production and has delivered in excess of $5.25 \mathrm{e} 12$ proton per pulse to the antiproton production target. The Main Injector cycle time for antiproton production is $1.46 \mathrm{sec}$.

Protons and antiprotons are accelerated in the Main Injector to $150 \mathrm{GeV}$ to fill The Tevatron for collider operation. In the process of proton acceleration, 5-7 bunches of protons are injected from Booster to the Main Injector in $53 \mathrm{MHz}$ buckets. The Main Injector accelerates these bunches using the $53 \mathrm{MHz}$ rf system to $150 \mathrm{GeV}$. At $150 \mathrm{GeV}$ the bunches are rotated by turning off the 53 $\mathrm{MHz}$ rf cavities and captured by $2.5 \mathrm{MHz}$ rf cavities to form a single high intensity coalesced bunch. Then the 53 $\mathrm{MHz} \mathrm{rf}$ is turned on and the beam is transferred to the Tevatron. This process is $85-92 \%$ efficient, with a bunch intensity of $250-330 \mathrm{e} 9 / \mathrm{bunch}$ and the longitudinal emittance is 2.2-3.2 eV-sec for 5-7 bunch operation. We routinely run the 7 bunch operation to meet the intensity demand of the Tevatron due to the poor injection and acceleration efficiency of the Tevatron. The longitudinal emittance of the proton beam is about 30\% higher than the Run-II goal, because of instabilities and the 7 bunches coalescing instead of 5 to meet the intensity demand.

The antiproton acceleration process in the Main Injector is similar to the proton process, except in this mode 4 batches of antiprotons (396 nsec apart) are injected from the Accumulator. One of the recent improvements in the Main Injector for the antiproton acceleration has been the implementation of multi-batch beam loading compensation. Figure 3 shows the longitudinal emittance of the antiprotons as a function of batch number for several stores. The emittance is larger for the higher bunch numbers due to beam loading. Figure 4 shows the same after the beam loading compensation has been commissioned. The average injected longitudinal emittance of the antiproton bunches is $1.5 \mathrm{eV}-\mathrm{Sec}$. There is a small growth during the acceleration process. As expected the coalescing process increases the longitudinal emittance by about a factor of 2 to an average value of about $2.7 \mathrm{eV}-\mathrm{Sec}$. The Run-II design goal of $2 \mathrm{eV}-\mathrm{Sec}$ was set assuming use of the Recycler and $2.5 \mathrm{MHz}$ acceleration. The $2.5 \mathrm{MHz}$ acceleration process in the Main Injector from $8 \mathrm{GeV}$ through transition energy to 27 $\mathrm{GeV}$ is designed to replaces the coalescing process. The

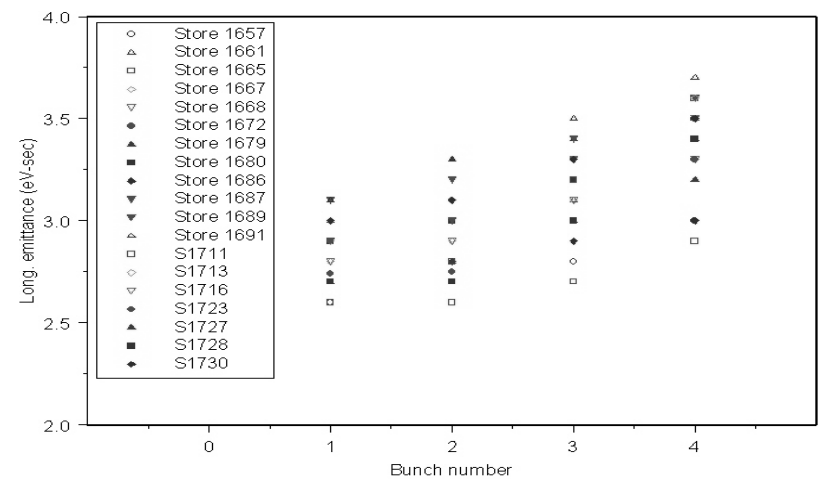

Figure 3. Longitudinal emittance of the antiproton as a function of bunch number with out beam loading compensation. 
average coalescing efficiency of the pbar acceleration and coalescing through the Main Injector exceeds $85 \%$. There is a small transverse emittance growth of less than $2 \mathrm{pi}$ $\mathrm{mm}-\mathrm{mr}$ (95\% normalized) in the Main Injector from injection to $150 \mathrm{GeV}$. The transverse emittance of the proton and antiproton beams in the Main Injector at 150 $\mathrm{GeV}$ is similar to Run-II design goals.

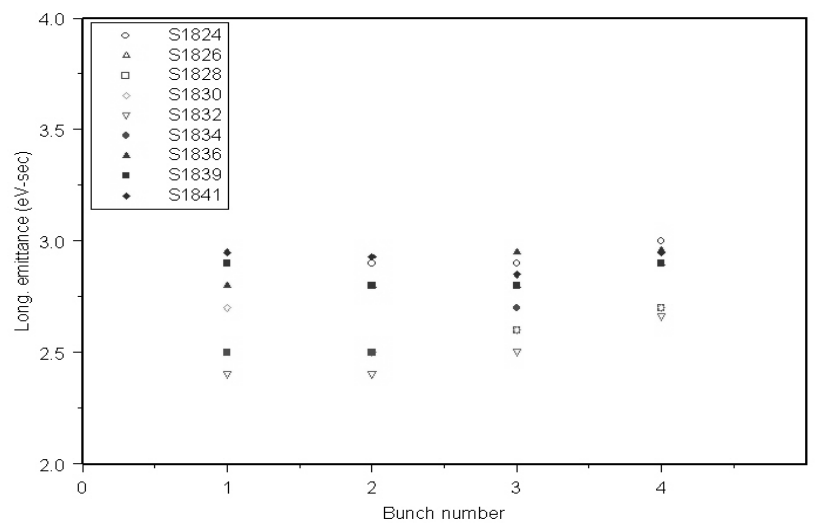

Figure 4. Longitudinal emittance of the antiproton as function of bunch number with beam loading compensation.

\section{ANTIPROTON SOURCE}

The Antiproton source was upgraded in association with the Main Injector project to increase the stacking rate to $18-20 \mathrm{e} 10$ antiprotons/hour [4]. A peak stacking rate of $13.5 \mathrm{e} 10$ antiprotons/hour and an average stacking rate of about $8 \mathrm{e} 10 / \mathrm{hr}$ have been achieved. The peak stacking rate is limited by the stochastic cooling, but the average stacking rate problem could be alleviated by storing antiprotons in the Recycler. One of the major problems with the antiproton source was the larger than expected transverse emittances of the cooled antiproton. Figure 5 shows the transverse emittance of the beam in Run-I and Run-II as a function of stack size. The typical horizontal emittance of a 1e12 antiproton stack was $17 \pi \mathrm{mm}-\mathrm{mr}$ as compared to the design goal of 8 . The larger emittance was due to an increased Intra-beam scattering (IBS) $(60 \%)$ caused by a change in the Accumulator lattice and trapped ions $(40 \%)$.

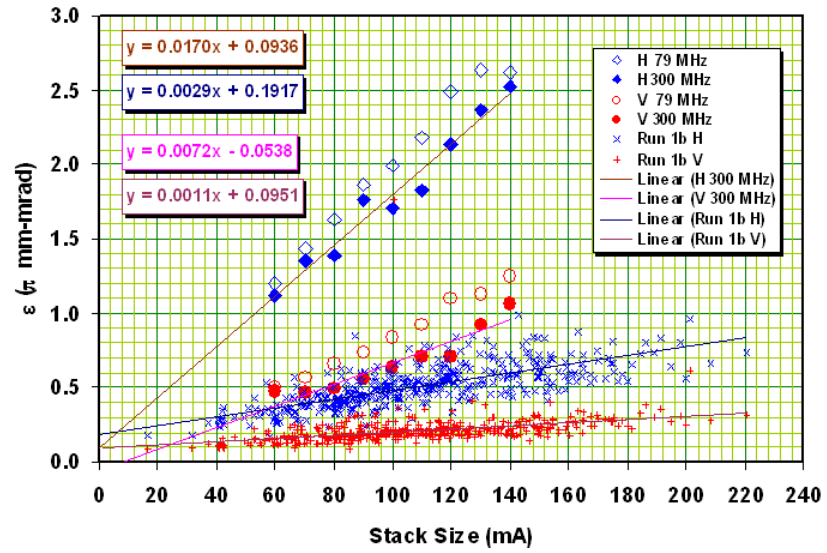

Figure 5. Accumulator core emittance as a function of stack size. These are unnormalized $95 \%$ emittances.
This large emittance problem was solved by a) better transverse stochastic cooling of the Accumulator core, and b) dual lattice operation of the Accumulator. The bandwidth of the stochastic cooling system was increased by a factor of two and the center frequency of the bean was increased by a factor of 1.5. Six new cooling systems and four new ultra-high vacuum tanks were built, installed and commissioned in less than 8 months. The dual lattice operation has a "fast stacking" lattice $(\eta=0.012)$ for pbar production and a "shot" lattice $(\eta=0.022)$ for Tevatron injection. During the shot setup for collider operation the Accumulator lattice is ramped with a cooled antiproton beam at the core orbit from fast stacking lattice to shot lattice. The shot lattice reduces the IBS heating by a factor of 2.5 and increases the cooling rate by a factor of 2 by increasing the mixing due to the change in $\eta$. Figure 6 shows the improvement in the emittance after these upgrade. These upgrades resulted in a factor of 2-3 reduction in core transverse emittance and a $40 \%$ increase in the collider luminosity.

The main Antiproton source R\&D efforts are towards increasing the stacking rate. The R\&D includes a) Debuncher momentum cooling improvements, b) a transverse Debuncher Notch Filter, c) commission core momentum stacktail compensation legs, d) implementing core momentum spreading during stacking, e) a stacktail notch filter upgrade, f) Improved transverse compensation of the stacktail, g) a reduction of Main Injector Longitudinal emittance, and h) an improvement of the AP2/Debuncher aperture

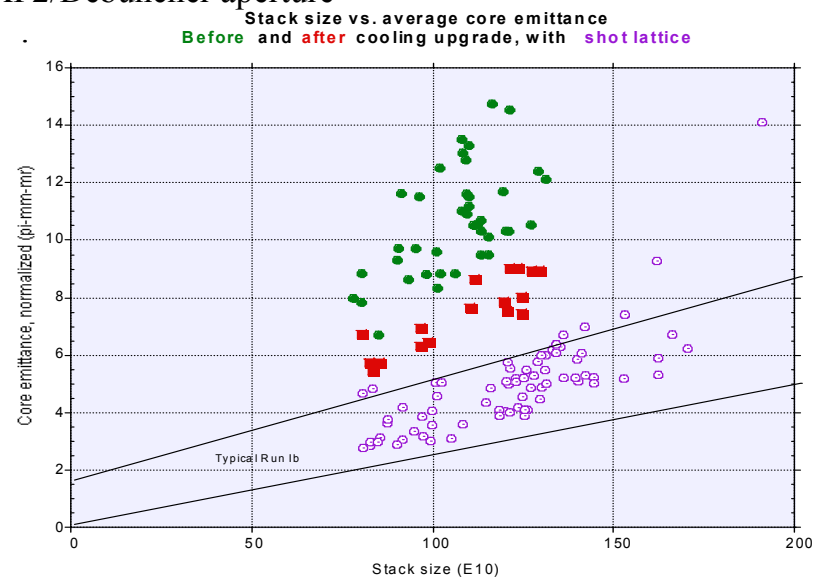

Figure 6. Average core emittance vs. Stack size. Green before upgrade, red after cooling upgrade and purple after shot lattice. ( $95 \%$ normalize emittance).

\section{TEVATRON}

The Tevatron has been making considerable progress in understanding the physics issues related to the delivery of higher luminosity [5]. It has been successful in utilizing the progress made in the upstream accelerator intensity and emittances and converted it to higher luminosity. Although a factor of two below the Run-II goals, the Tevatron is typically running with a factor of two higher peak luminosity than Run-I. This situation is due to 
fundamental physics limitations, which are new compared to Run-I, such as larger beam-beam effects, instabilities and poor lifetime. We are running with higher number of bunches $36 \times 36$ as compared to $6 \times 6$. There are several limitations in understanding and solving these issues, including poor BPM, unstable Tevatron Lattice due to alignment and orbit motion and lack of beam study time.

Figure 7 shows typical beam intensities for a Tevatron collider fill. We observe a loss of proton beam at injection, open of helix and poor lifetime at $150 \mathrm{GeV}$. The pbar beam, although $1 / 10$ the intensity of the protons, has

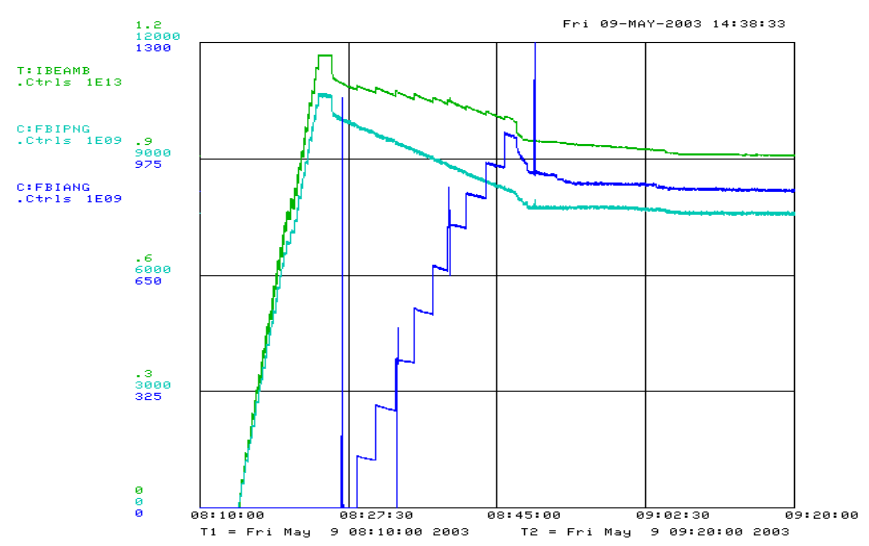

Figure 7. Typical Tevatron fill intensity and losses. Green is the total proton intensity, sky blue is the bunched proton beam intensity and blue is the bunched antiproton.

a poor lifetime due to larger beam growth at injection into the Tevatron and the stronger beam-beam effect. About $10 \%$ of the bunched beam is lost during the ramp and squeeze for collision.

Several controlled beam studies have been performed to study and improve the performance of the Tevatron. These improvements include a) tune, coupling and chromaticity compensation at $150 \mathrm{GeV}$ through collision, b) orbit smoothing, c) a longitudinal damper to stop $\sigma_{\mathrm{s}}$ blowup during store, d) a transverse damper to improve $150 \mathrm{GeV}$ lifetime, and e) general tuning of the machine.

The beam-beam effect is one of the main contributions to the poor lifetime of the pbar beam in the Tevatron. Several observations indicated that a smaller pbar beam size yields a better pbar lifetime at $150 \mathrm{GeV}$ and that an antiproton only store has a better lifetime. During the Jan 03 shutdown the Lambertson at the $\mathrm{C} 0$ region was replaced with Main Injector dipoles to increase the helix separation and to reduce the impedance of the Tevatron. We are also investigating the possibility of improving diagnostic in the Tevatron (new BPMs electronics, Schottky etc.), including improvements in hardware and understanding systematic errors. Several study groups have been formed to systematically study Tevatron beam dynamics.

\section{RECYCLER}

The Recycler is designed to store and cool antiprotons at $8.9 \mathrm{GeV} / \mathrm{c}$. It was built using permanent magnet technology, but during the commissioning period ramped dipole correctors were added. In its first phase, the Recycler was designed to store $2 \mathrm{e} 12$ antiproton by frequent transfer of antiprotons from the Accumulator to the Recycler. The Accumulator stacking rate decreases as the stack size in the Accumulator increases. The plan is to transfer antiprotons from the Accumulator to the Recycler every 2-3 hours to keep the stacking rate high. The design goal of the Recycler Ring is to have $2 \mathrm{e} 12$ antiprotons stacked with 100 hours of lifetime using stochastic cooling. At a later date, the Recycler cooling will be upgraded with electron cooling.

The Recycler Ring has made considerable commissioning progress over last two years [6]. Fig. 8 shows the stacking of antiprotons in the Recycler Ring with total efficiency of $75 \%$. The best lifetime achieved for pbars is 180 hours. The lifetime has a small dependence on the stack size in the Recycler: lifetime $=$ $0.44 x($ Stack Size $)+183$. We have the mechanisms in place to transfer pbars from the Accumulator to the Recycler, cool the pbar in the Recycler, extract and bunch the pbars in 2.5 Mhz bunches from the Recycler, transfer the pbar bunches to the Main Injector, accelerate the pbars in the Main Injector to $150 \mathrm{GeV}$, and transfer them to the Tevatron.

The Recycler needs improvements in its performance before it can be integrated into the Accelerator complex. Some of the issues are a) injection and circulating efficiency for proton and pbar is about 90-95\%, b) emittance growth is about a factor of 2 larger than the design value, c) the longitudinal emittance is effected by the Main Injector ramp and is larger than the design goal, d) the Recycler orbit is not well established due to lack of a working BPM, e) cooling and RF manipulations needs further optimization.

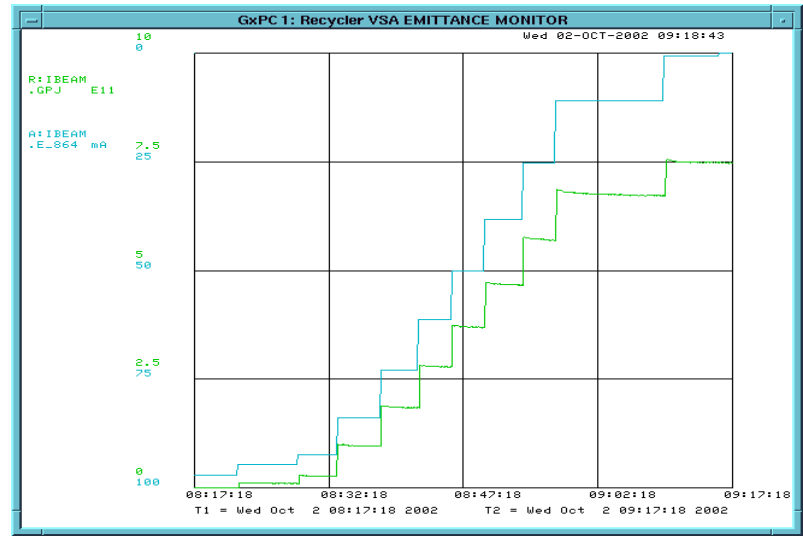

Figure 8. Stacking of pbar in the Recycler. Blue in the pbar intensity in the Accumulator and Green in the Recycler intensity. The scale of Blue is reversed.

A significant fraction of Recycler beam transverse emittance growth is vacuum related. A plan is in place to improve the Recycler vacuum by doubling the number of ion pumps, baking the Recycler to a higher temperature and fixing small leaks, which may be present around the ring. The ramped correctors will be used to reduce the effect of the Main Injector ramp on the Recycler. Initial 
tests already show positive results. The Recycler BPM electronics are being upgraded using digital electronics. It is expected that with the upgrade and integration plans that are already in place the Recycler will perform as described in its initial design report.

\section{COLLIDER PERFORMANCE}

In the last one year the luminosity of the Tevatron collider has improved by more than a factor of 3 . The collider luminosity is within a factor of two of the Main Injector design. Every improvement in luminosity of the complex has been associated with a specific modification to the accelerators. These included a) Accumulator cooling and shot lattice $(40 \%)$, b) Accumulator to Main Injector lattice match and antiproton emittance at Tevatron injection $(20 \%)$, c) antiproton coalescing improvement in the Main Injector (15\%), d) Improved antiproton and proton efficiency through Tevatron low beta $(10 \%$ each), and e) larger antiproton stack $(10 \%)$. Most of the luminosity increase is due to increasing the number of antiprotons at low beta. Figure 9 shows the total number of antiproton to low beta as a function of shot number.

The current antiproton production rate is sufficient to support a luminosity in the $6-8 \mathrm{e} 31 \mathrm{~cm}^{-2} \mathrm{sec}^{-1}$ range. The protons at low beta are $95 \%$ of the goal. Although antiproton efficiency has improved from $32 \%$ to $70 \%$, the antiprotons are still only $70 \%$ of the goal. The primary outstanding issues towards achieving higher luminosity are a) emittance preservation from Tevatron injection to acceleration in Tevatron, b) proton and antiproton lifetime and acceleration efficiency in the Tevatron and c) Tevatron beam dynamics which is adversely effected by beam-beam effects, lattice, alignment and coupling. A variety of hardware projects are currently underway dealing with these issues. An upgrade plan is being developed to improve the luminosity even further.

Figure 10 shows the weekly peak luminosity as a function of week number and peak luminosity as a $10 \mathrm{x}$ running average. The luminosity has been increasing steadily since the beginning of Run-II. At the time of this talk the Accelerator complex has delivered in excess of $220 \mathrm{pb}^{-1}$ to each detector since the beginning of the run.

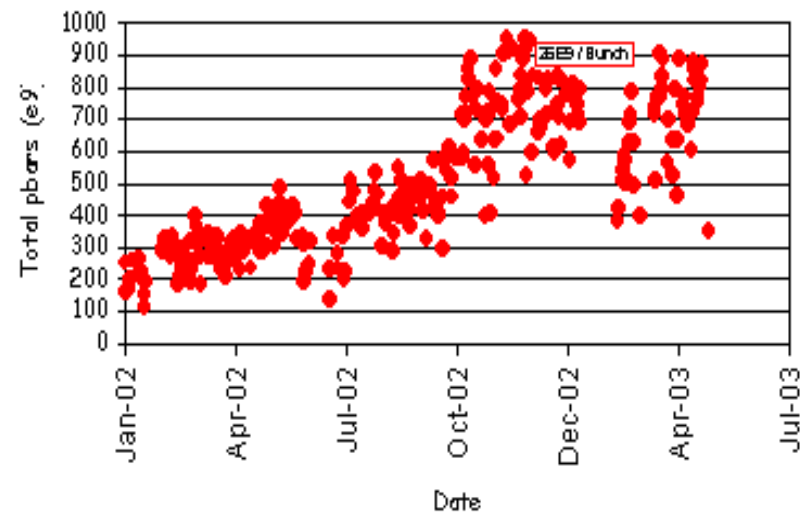

Figure 9. Total number of antiproton at low beta.

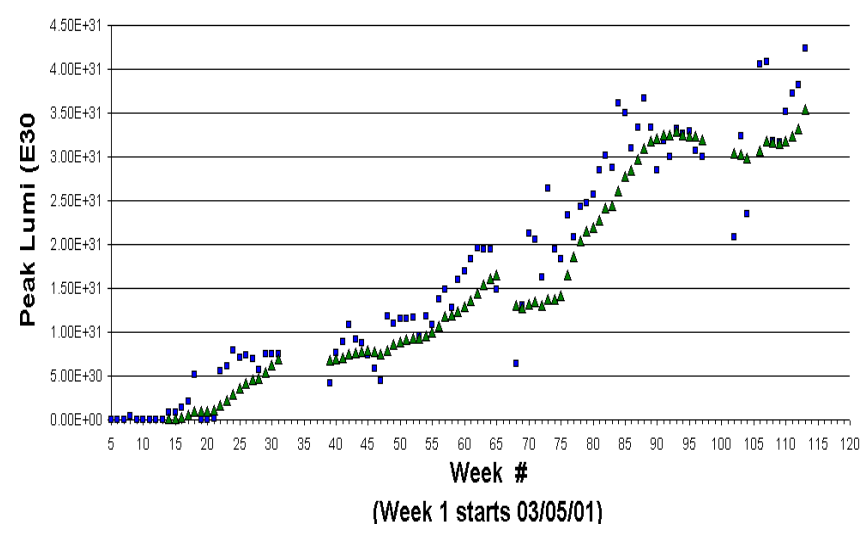

Figure 10. Peak Luminosity as a function of week. Blue is the peak luminosity of the week and green the 10 shot running average.

The goal of the collider program for FY03 is to deliver $200 \mathrm{pb}-1$, with a peak luminosity of $10 \mathrm{pb}-1$ per week by the end of FY2003.

\section{SUMMARY}

The Fermilab collider program at $1.96 \mathrm{TeV}$ is well underway with exciting physics results from both the collider detectors. The collider is running with in a factor of two of the Run-II designed luminosity with the new Main Injector. There are several hardware upgrade projects underway to improve the luminosity. We are interleaving accelerator studies and operations to further improve the luminosity. The Recycler Ring commissioning has progressed well and the machine is close to being ready for integration into the accelerator complex after a shutdown to improve vacuum and instrumentation.

\section{ACKNOWLEDGEMENT}

I will like to thank the following for helping me prepare this talk by providing their talks and discussing the details of their view of accelerator complex with me; Elliot McCory, Dave Johnson, Elvin Harm, Mike Martin and Steve Holmes. I will also like to thank John Marrier for his valuable comments on the talk and paper.

\section{REFERENCES}

[1] E. McCory, talk at AAC : http://www-bd.fnal.gov/aac/ Fermilab, Feb 2003.

[2] W. Chou et al. 'Fermilab Booster Modeling and Space Charge studies.', PAC2003 Proceeding, Portland.

[3] D. Johnson, talk at AAC http://www-bd.fnal.gov/acc/ Feb 2003, Fermilab.

[4] E. Harm, talk at AAC. Feb 2003, Fermilab.

[5] M. Matrin, talk at AAC, Feb 2003, Fermilab.

[6] S. Mishra, talk at AAC, Feb 2003, Fermilab.

*Work supported by the US department of energy under contract DE-AC02-76CH003

\#Email: Mishra@fnal.gov 